\title{
Psychiatric advance directives: qualitative study of informed deliberations by mental health service
}

\section{users}

\author{
MICHAELA AMERING, PETER STASTNY and KIM HOPPER
}

\author{
Background Established legal \\ mandates and high expectations for \\ psychiatric advance directives are not \\ matched by empirical evidence \\ documenting their actual implementation.
}

Aims To explore the interests, concerns and planning activities of informed mental health service users contemplating such directives.

Method Standard qualitative research techniques were used: field observations, interviews, focus groups, archival research and key informant interviews; 33 persons participated in the interviews and focus groups. Transcripts were coded and analysed for thematic content, and results were member-checked.

\section{Results Training set in motion labour- intensive projects: conceptualising how a psychiatric advance directive would work in one's life, mobilising resources, reviewing past experiences and assessing risks. Especially meaningful was the prospect of being treated as a responsible agent in future interactions with the mental health system.}

\section{Conclusions Advance directives are} best thought of as complex planning tools for future psychiatric crisis management, rather than focal interventions to enhance compliance. Research is needed to explore the institutional response to this prospective decision-sharing initiative.

Declaration of interest None.
Psychiatric advance directives are legal instruments that allow competent individuals to appoint proxies and specify how treatment decisions should be made in the event they become incompetent. These directives have been hailed as instruments for honouring patient autonomy, improving communication and ensuring participation in treatment decisions, and providing pragmatic alternatives to coercive measures (Appelbaum, 1991; Swanson et al, 2000; Srebnik et al, 2003). Progress towards implementing advance directives as routine planning options has not kept pace with such enthusiasm.

The only randomised effectiveness study of psychiatric advance directives showed no impact on outcome of care with regard to compulsory admissions, hospital days or patient satisfaction (Papageorgiou et al, 2002). However, as with other complex community-based interventions (Schoenwald \& Hoagwood, 2001; Ablon \& Jones, 2002; Hohmann \& Shear, 2002), questions have been raised about actual operational specifics and how they might have influenced the impact of local efforts to implement such directives (Thomas, 2003).

No scientific consensus exists on what the 'intervention' is. It is unclear what it means for people with severe and persistent mental illness to undertake the anticipatory planning culminating in an advance directive. This study empirically explores the deliberations of informed mental health service users contemplating this option.

\section{METHOD}

Long accepted as a productive partner in public health and evaluation research, qualitative research methods have begun to proliferate in health services research (Devers et al, 1999), clinical studies (Miller \& Crabtree, 1994), health technology assessment (Murphy et al, 1998) and community-based intervention research (Hohmann \& Shear, 2002). Such methods are preferred in field settings where the scope of work has yet to be determined, the relevant questions have still to be precisely formulated, local understandings are in flux and institutional arrangements unsettled (Buston et al, 1998). Such conditions aptly describe the state of patients' training in psychiatric advance directives in New York State late in 2001, when this study commenced.

Formal training in psychiatric advance directives, by the user-run Resource Center, had begun in May 2000. Up to five educators crisscrossed the state, offering intensive sessions, working with official healthcare proxy forms, to recipients of public mental health services. By January 2002 (when recruitment for this study ended), the programme had logged 279 training sessions statewide, involving nearly 6000 participants.

\section{Data collection}

Standard qualitative techniques were employed. Field observations were made over a period of 6 weeks by one of the authors (M.A.), who attended eight training sessions. These sessions also served as recruiting sites for potential interviewees and focus group participants. Archival research was undertaken to locate and review relevant legislation and memoranda, annual reports of the training programme and interim evaluations of its work. Key informant interviews were conducted with the training programme director, three trainers, state officials, legislative aides, advocates and peer specialists. Extensive open-ended interviews were conducted by one or two researchers (M.A. and K.H.) with 20 individuals who had received training in the preparation of advance directives at one of three different psychiatric rehabilitation programmes. The aim of our study was to interview ten people who had decided to compile a psychiatric advance directive after participating in the training and ten people who had declined to do so. The latter were recruited from among 88 of 270 trainees who had expressed interest in participating in the study, as were three of those who had decided to draft an advance directive; the other seven people in the advance directive group were recruited through snowball sampling. Another 13 people were recruited for two focus groups. All 33 participants (Table 1) 
Table I Study participants

\begin{tabular}{lcc}
\hline & $\begin{array}{c}\text { Interview } \\
\text { group }\end{array}$ & $\begin{array}{c}\text { Focus } \\
\text { group }\end{array}$ \\
\hline $\begin{array}{l}\text { Gender, } n \\
\text { Women }\end{array}$ & 12 & 4 \\
Men & 8 & 9 \\
Ethnicity, $n$ & & \\
White & & \\
Women & 8 & 1 \\
Men & 3 & 4 \\
African-American & & \\
Women & 3 & 1 \\
Men & 3 & 4 \\
Hispanic & & \\
Women & 1 & 2 \\
Men & 1 & 1 \\
Asian-American & & 0 \\
Women & 0 & $25-60$ \\
Men & 1 & \\
Age range, years & & \\
\hline & & \\
\hline
\end{tabular}

had extensive experience with mental health services and crisis interventions; most had been hospitalised on several occasions for severe mental illness. Interviews lasted $1-6 \mathrm{~h}$ and could take two sessions to complete; the focus groups ran for $1 \frac{1}{2} \mathrm{~h}$. Participants were invited to 'think out loud' as they reflected on the training and recalled their subsequent reasoning and actions vis-à-vis psychiatric advance directives. All sessions were tape-recorded and transcribed. Finally, preliminary results underwent provisional member-checking in subsequent discussions with the training project director and six study participants.

After the study had been fully explained, all participants gave written informed consent to the taping of all communications, analyses of transcripts and publication of results and anonymous quotes. Informed consent forms had been approved with the study plan by the New York State Office of Mental Health's Institutional Review Board.

\section{Analysis}

Transcripts of interviews and focus group meetings were coded for content following prescribed conventions (Miles \& Huberman, 1994; Emerson et al, 1995; Ryan \& Bernard, 2000). Data collection (interviewing) and initial analysis (coding) proceeded in a linked fashion. All three investigators closely read transcripts of the initial interviews, identifying topics of interest (open coding) either because they pertained to issues already flagged in the literature (e.g. comprehension, illness and identity, coercion) or because they emerged in the interview as issues of vital concern to the participant (ensuring respect, patching system gaps in record-keeping, customising treatment). These separate readings were then compared and extensively discussed, and a synthetic set of codes was constructed to guide both the conduct of the next interviews and analysis of their transcripts. This process was repeated, modifying the working codebook and recoding earlier sets of transcripts in a directed fashion (focused coding), then testing the most recent codes on fresh transcripts, until a final set of 10 codes (see Appendix) and 24 sub-codes was arrived at; in this way, 'saturation' was reached, at which point new data could be accommodated by existing categories. This coding protocol was systematically applied to the entire corpus of transcribed text using software (QSR-N6) to facilitate marking and retrieval of segments. The substantive groupings of text (e.g. knowledge of advance directives; feasibility worries; family and friends) were examined and 'interrogated' so that higher-order themes concepts (Becker, 1998), schemata (Agar \& Hobbs, 1985) and low-level 'theories' (Strauss \& Corbin, 1990) - might be analytically derived. This process was aided by working memoranda that set down provisional interpretations or hypotheses about what we had witnessed. In this way, we attempted to map the contours (or cultural domains) of a still-evolving practice: the implicit rules, organising anxieties, defined tasks and unresolved tensions at stake in these attempts to reckon with the prospects of psychiatric advance directives (Spradley, 1980).

\section{RESULTS}

Where questions of meaning are vital, varied and contested, qualitative methods serve as exploratory tools. Even commonsensical notions are best approached sceptically. We soon learned, for example, that it would be necessary to rethink the 'decision' to execute an advance directive. Interest, understanding, readiness, follow-through, problem-solving and persistence all took shape as complex embodiments of history, present circumstance and informal risk assessment. Executing an advance directive, it became clear, is a project: a sustained act of self-examination and situation appraisal.

After describing the training process, we report results under two headings: the work involved and the meanings construed. To anticipate somewhat: it was the deliberating process itself $-\mathrm{a}$ process that can all too conveniently be 'black-boxed' in simple outcome studies-that emerged as the defining feature of this inquiry.

\section{Training}

Eight workshops, involving one or two trainers and 10-100 participants, were observed. All took place as a part of the regular rehabilitation curriculum at participating sites. Written material and actual forms were distributed, a detailed runthrough of the process was conducted and questions actively solicited. The last typically concerned the legal and administrative basis of psychiatric advance directives, their scope and limitations, procedures for revising or revoking them and details regarding appointing an agent. Most of those attending the workshops clearly understood what the directives were about and endorsed their intent. Discussion of probable logistical complications (e.g. lack of a central registry), however, raised doubts about the feasibility of these directives.

\section{Work}

\section{Grasping the concept}

Interviewees showed no difficulty in understanding the concept of psychiatric advance directives. The syntax might have been irregular at times - 'It means you have to go back to the word itself, it means advance is thinking forward, in a moment of center and lucidity and being directive' - but, as subsequent discussion confirmed, the meaning was clear. Several interviewees and focus group members were already familiar with healthcare proxies through prior experience with kin or friends. Others had learned about them before the training from consumer advocates or presentations in mental health settings.

\section{Imagining directives in one's own life}

Notwithstanding the intuitive appeal of psychiatric advance directives, committing to the process that would culminate in a fully executed directive proved difficult 
because it meant individualising what one would entail. Participants were drawn to the concept because their wishes would be more likely to be honoured in situations in which they had felt powerless in the past. A designated agent and documented preferences would improve record-keeping and communication in a system widely seen as inadequate in both.

Participants imagined trusted agents undertaking interpretation and negotiation on their behalf: articulating their wishes, translating what clinicians were saying and responding in accordance with the participant's preferences. Directives held the promise that participants would not have to explain everything yet again, that they would be believed without having to persuade strangers, that confrontations could be avoided through judicious intervention by their proxy and that appropriate treatment would be expedited. Instead of fruitless and increasingly furious exchanges with hospital personnel, one participant imagined producing a card with her proxy's name and stepping back to let negotiations proceed in a calm and orderly fashion.

No participant viewed advance directives as a blanket means to refuse treatment. None seized upon it to make a point or lodge a grievance. Without exception, the participants were concerned chiefly with improving their treatment, ensuring that their own experience would become part of the 'evidence base' on which future decisions would be predicated. The directives were designed to inform clinical teams, and a great deal of thought went into enhancing their instructive value. Interviewees were hopeful, too, that having a directive would alleviate the burden on others by assuming responsibility for treatment in advance of crises.

Imagining advance directives in action could also produce reasons for avoiding them. One interviewee feared that executing a directive could actually invite the situation it was designed to manage. For others, pragmatic issues dominated: 'more paperwork, more hassles'; concerns that the directive might not 'stand up in court' or would be overridden in practice. For many, past experience counselled scepticism, if not outright pessimism: in their minds, the mental health system could hardly be trusted to reform merely because of this 'legalistic' tool. Such doubts could be disabling, derailing the deliberation process early on.

\section{From concept to labour}

Finding oneself intrigued by the notion and personalising the concept of an advance directive does not yet commit one to the necessary work of executing it. A number of catalysts can be identified in the accounts of participants who took the step. These tended to operate in cumulative fashion, acquiring persuasive force over time (only a few participants initiated the process soon after the training). Further discussions with mental health professionals clearly motivated some to proceed. Others had been asked about advance directives during their last stay in hospital and now had occasion to act. Many were nudged by terminal illness or psychiatric hospitalisations affecting their families or partners. For others, a reconfigured personal network supplied previously missing others who could be trusted to serve as proxies.

\section{Reviewing past experience}

An essential part of the labour was a systematic review of past experiences with psychiatric crises, hospital admissions and medication history. Rehearsing the past, we were repeatedly told, is an exercise fraught with trepidation and pain-but necessary to 'make sense of your own history'. Contemplating an advance directive can reframe a psychiatric history as a resource from which valuable guides to future system involvement might be gleaned. It can also be risky: memories of traumatic experiences (psychotic crises or difficult treatment situations) can be both upsetting and feared as possible triggers of a new episode. This reinforces the impression that the drafting of advance directives may need to proceed according to the individual's own timetable, as service users work through their personal risk-benefit ratios.

\section{Mobilising resources}

Executing an advance directive means negotiating a host of practical difficulties: securing the technical assistance to complete the documentation, filing the requisite copies at appropriate sites, notifying key collaborators and setting up procedures for periodic review. Appointing the agent proved the decisive issue. Family members were typically the first option, with siblings and aunts or uncles often preferred to parents (seen as too close to serve as advocate for the individual's wishes). Some candidates were eliminated because the responsibility 'would be too hard on them' or because they might be unable to act with the requisite discretion. Peers were considered, together with concerns that they might not be seen as competent by professionals. Some believed that appointing a mental health professional would give them a distinctive edge. A number were disappointed to learn that their own therapists refused because of possible conflict of interest. Even when appropriate candidates were identified, some were reluctant to approach them, fearing rebuff.

\section{Completing the process}

Respondents varied greatly in the duration of decision-making. Again, few executed a directive in the immediate aftermath of training. Others took years to mobilise the resources and muster the courage to see the process through. For one interviewee, it had taken a decade-long, wholesale reconfiguration of her personal life before the trusted others were in place. It was not unusual for several versions to be discarded before a satisfactory directive was completed. In all but one executed, the process included appointing an agent.

Although not a specific goal of this study, we did learn much about the content of psychiatric advance directives. Apart from preferences for certain hospitals or specific professionals, requests to allow favoured coping strategies (such as being left alone at times or listening to music) and boundary rules (such as not being touched by staff without being asked) or to proscribe certain drugs or treatments (such as electroconvulsive therapy) were among the detailed instructions. Favoured medications were also named and dosages specified. Reasons for choices, drawn from past experience with ineffectiveness or unwanted side-effects, were documented. Some listed people whose company they preferred and others whose presence they could do without. Much thought was given to ensuring that the advance directives were feasible and that preferences fell reasonably within the range of options of the mental health system. Participants were intent on not compromising mental health professionals with either the content or the style of the directive. It was widely assumed that an ill-advised set of instructions for the appointment of an overly aggressive advocate could backfire, provoking resentment 
and non-compliance on the part of healthcare staff.

\section{Meaning \\ Taking on responsibility}

Committing to the work of drawing up an advance directive was demanding and consequential. For many, this meant accepting a new order of responsibility for managing their illness, containing the trouble crises might cause, securing the allegiance of essential others and thinking through what would constitute a productive response from mental health professionals. It meant taking oneself seriously as an actor in the orchestration of one's treatment, not merely being its object or victim.

\section{The ordeal of looking ahead - and back}

Anticipatory planning for psychiatric emergencies is both taxing and unnerving. Undertaking a searching inventory of past experience and present-day resources, facing up to 'the possibility of losing capacity' and the humiliation it so often entails, taking pre-emptive action to forestall gratuitous harm and ensure interventions of proven benefit, and asking someone to assume the role of agent: this takes courage and perseverance. Effectively taking the process through to completion was a source of great satisfaction and pride for the participants.

\section{A doubly instructive effort}

Psychiatric advance directives provide a means of coming to terms with the exigencies of living with severe mental illness and understanding how others figure in that story. Their benefits additionally extend to critically positioned future readers of these directives - mental health professionals in crisis settings - whose knowledge of the directive's author would be substantially enhanced.

\section{Holding on to one's identity}

Making formal arrangements for one's true self to be present, consulted and represented by a surrogate was a much-prized feature of psychiatric advance directives. The force of the document ('that they would have to honour it') resided in its power to transmit the intentions of a self whose capacity calmly to articulate its interests could become degraded at times. In the hectic circumstances of psychiatric crises, the directive would contain and project a corrective competence, stemming the inertial process of becoming 'just a psychiatric patient'. Here was written evidence that people had 'thought enough of themselves before coming to the hospital that they put some thought into this'. That made it a 'thing of respect'.

\section{A new form of cooperation}

Advance directives serve not only a protective purpose (as a 'shield' and 'buffer') but a collaborative purpose (as a 'contract') as well. In exchange for stipulating their preferred terms and conditions for receiving care, individuals pledge to work with the system, negotiating problems through the intervention of an appointed intermediary.

\section{DISCUSSION}

Reframing our research concern as deliberation rather than decision with respect to psychiatric advance directives reflected the actual reality of a continuum of interest and action. With few exceptions, those who already had executed an advance directive had undertaken the difficult work that the still-undecided participants were actively engaged in.

\section{Discordant cases}

Even on their own terms, qualitative methods are incomplete if they fail to account for discordant cases in the textual evidence amassed. In one departure from the pattern, a long-term veteran of the mental health system saw advance directives as completely irrelevant in his own case, but did endorse them for others as protection against abuse he had witnessed. A second exception, occasionally voiced in training sessions, should also be mentioned: the stance of people with so little trust in the system that the prospect of undertaking the work of preparing a directive seemed no more than an elaborate joke.

\section{Limitations of the study}

Although the small sample size and interview time frame are limitations, the exploratory nature of this study should be stressed. Within the limits of experience to date, this group of informants proved willing and able to 'think out loud' about the whole process with researchers in attendance, rehearsing anxieties, misgivings, hopes and their own peculiar brands of risk-benefit accounting. Sufficient resonance across the group was reached that we think it safe to say that the themes emerging in these labour-intensive planning projects are worth pursuing with more rigorous and protracted methods of investigation.

\section{Interest v. follow-through}

Our findings are congruent with other studies, cited earlier, that documented high levels of interest in psychiatric advance directives among people with a diagnosis of severe mental illness, their families and providers (Amering et al, 1999; Backlar et al, 2001; Swanson et al, 2003; Srebnik et al, 2003). We, too, found that most people easily understood the concept of advance directives and were uninterested in using them to refuse all treatment (Sherman, 1998; Backlar et al, 2001; Valletto et al, 2002). Given the well-documented obstacles to implementing advance directives lack of information and support for staff, legal uncertainties, want of ongoing assistance and administrative indifference (Howe, 2000; Srebnik \& Brodoff, 2003) - the low rates of completion of such directives are not surprising. Discordance between interest and follow-through is not uncommon in studies of preparatory planning: for example, only $16 \%$ of healthy, motivated relatives of people with Alzheimer's disease returned a self-addressed stamped envelope to ratify an earlier willingness to participate in research (Wendler et al, 2002).

\section{Advance directives as projects}

We found the space between expressed interest and formal execution to be a patchwork of industry. Queried about their thinking and activity after the training sessions, study participants talked about work: sometimes inefficient, occasionally stalled, usually protracted and operating at variable pace. Although a few were flummoxed by the scale of the project, most were not only ready to engage in constructive thinking about their directives, but also willing to take on more responsibility for treatment preparedness than ever before. This meant substantial activity and personal risk. What sustained the work and made the risks worth taking was the promise of a new relationship with the mental health system, one built on collaboration and respect for their own experience-based expertise. Accordingly, people were careful 
to structure their directives so that professionals could respond constructively.

\section{System receptivity}

As psychiatric advance directives slowly make their way into the clinical landscape, the mental health system faces a formidable challenge. If it is not met, the offer of advance directives risks becoming a charade, instead of the good-faith invitation to dialogue that most of our informants interpreted it to mean. Implementing new procedures, even ones acclaimed in principle, is invariably experienced as cumbersome and artificial at the outset. A good deal of trial and error is needed before workable, culturally congruent practices are arrived at. Our findings suggest that sufficient deliberative time, and the resources to support and inform decisionmaking, will be crucial to the implementation of advance directives. 'Pushed timing' undermines voluntarism, degrades capacity to make a considered choice tailored to one's life circumstances and values, and nullifies the ethic of respect that motivates shared decision-making (Roberts, 2002). Peer advocates may well be the ideal training mentors (Backlar et al, 2001), but the protracted and unpredictable nature of deliberation makes a standing offer of technical support advisable.

Psychiatric advance directives are best conceived as 'complex interventions that require lengthy development work if they are to stand a chance of success' (Thomas, 2003). The development work includes not only patients, family members and clinicians, but also the system itself. A host of legal, procedural and logistical problems, well described in the literature on healthcare proxies (Slewchuk, 1998), remain to be addressed. Resolving them will require substantial investment of resources to effect the necessary changes in administrative infrastructure and professional culture, at a time when the moral economy of care appears to be heading in a number of contrary directions (Hopper, 2001). Advance directives hold great potential to engage consumers in a serious and balanced collaborative process, but without a serious systemic commitment this bold experiment may prove a cautionary tale instead.

\section{ACKNOWLEDGEMENTS}

This research was funded by the Center for the Study of Issues in Public Mental Health, based at

\section{CLINICAL IMPLICATIONS}

- Understanding how (and how well) psychiatric advance directives work will require close documentation of the processes involved in implementing them.

- Intensive training and attentive follow-through would greatly aid the deliberating activities of people contemplating executing psychiatric advance directives.

Substantial administrative commitment on the part of the mental health system is the necessary complement to expressed patient interest and preparatory action.

\section{LIMITATIONS}

Findings of our study may not be generalisable because the sample size was small and participants were not chosen randomly.

Full documentation of deliberations over advance directives would have required interviews and observations over a longer period, as the directives were tested and changes made.

- Not enough people who had decided against executing an advance directive were interviewed to determine how they differed from those actively considering one.

MICHAELA AMERING, MD, Department of Psychiatry, University of Vienna, Austria; PETER STASTNY, MD, Bronx Psychiatric Center, New York; KIM HOPPER, PhD, Nathan S. Kline Institute for Psychiatric Research, New York, USA

Correspondence: Kim Hopper, Nathan Kline Institute, 140 Old Orangebury Road, Orangebury, New York,USA; e-mail: hopper@nki.rfmh.org

(First received 16 December 2003, final revision 16 September 2004, accepted 29 September 2004)

Nathan S. Kline Institute for Psychiatric Research, Orangeburg, New York, and by ongoing support of two of the investigators (P.S. and K.H.) by the New York State Office of Mental Health. The authors thank Melanie Shaw for extensive discussions of earlier drafts.

\section{APPENDIX}

\section{Text coding categories}

\section{Knowledge}

Informant's knowledge about advance directives, whether gained through training or experience.

\section{Feasibility}

Concerns about the logistics of advance directives, including legal status, mechanics of use and the system's capacity to respond.

\section{Experience}

Experience with the mental health system - positive and negative - and especially with coercion.

\section{Anxiety}

Fears raised with respect to drafting an advance directive thinking about past, future, oneself as agent.

Intent

Changes the informant hopes to make through the vehicle of an advance directive.

\section{Unintended changes}

Changes the informant fears might ensue because of an advance directive.

Content

Actual content of an advance directive.

\section{Action}

Comments relating to deciding on or taking action, or barriers to action, in drafting an advance directive. 


\section{Editorial}

Expressed opinions, ideological statements.

\section{Uncoded}

Material not otherwise coded

\section{REFERENCES}

Ablon, J. S. \& Jones, E. E. (2002) Validity of controlle clinical trials of psychotherapy: findings from the NIMH Treatment of Depression Collaborative Research Program. American Journal of Psychiatry, I59, 775-783.

Agar, M. \& Hobbs, J. (1985) How to grow schemata out of interviews. In Directions in Cognitive Anthropology (ed. J.W. Dougherty), pp. 4I3-43I. Urbana: University of Illinois Press.

Amering, M., Denk, E., Griengl, H., et al (1999) Psychiatric wills of mental health professionals: a survey of opinions regarding advance directives in psychiatry. Social Psychiatry and Psychiatric Epidemiology, 34, 30-34.

Appelbaum, P. S. (1991) Advance directives for psychiatric treatment. Hospital and Community Psychiatry, 42, 983-984.

Backlar, P., McFarland, B. H., Swanson, J.W., et al (200I) Consumer, provider, and informal caregive opinions on psychiatric advance directives.

Administration and Policy in Mental Health, 28, 427-44I.

Becker, H. S. (1998) Tricks of the Trade: How to Think About Your Research While You're Doing It. Chicago: University of Chicago Press.

Buston, K., Parry-Jones, W., Livingston, M., et o (1998) Qualitative research. British Journal of Psychiatry, 172, 197-199.

Devers, K. J., Sofaer, S. \& Rundall, R. G. (1999) Qualitative methods in health services. Health Services Research, 34 suppl. (no. 5, part II, monograph).
Emerson, R. M., Fretz, R. I. \& Shaw, L. L. (1995) Writing Ethnographic Fieldnotes. Chicago: University of Chicago Press.

Hohmann, A. A. \& Shear, M. K. (2002) Community based intervention research: coping with the 'noise' of real life in study design. American Journal of Psychiatry, I59, 20I-207.

Hopper, K. (200I) Commentary: on the transformation of the moral economy of care. Culture, Medicine and Psychiatry, 25, 473-484.

Howe, E. G. (2000) Lessons from advance directives for PADs. Psychiatry, 63, 173-177.

Miles, M. \& Huberman, M. B. (1994) Qualitative Data Analysis: An Expanded Sourcebook. Newbury Park, CA: Sage.

Miller, W. L. \& Crabtree, B. F. (1994) Qualitative analysis: how to begin making sense. Family Practice Research Journal, 14, 289-297.

Murphy, E., Dingwall, R., Greatbatch, D., et al (1998) Qualitative research methods in health technology assessment: a review of the literature. Health Technology Assessment, 2, I-274.

Papageorgiou, A., King, M., Janmohamed, A., et al (2002) Advance directives for patients compulsorily admitted to hospital with serious mental illness: randomised controlled trial. British Journal of Psychiatry, I8I, 513-519.

Roberts, L.W. (2002) Informed consent and the capacity for voluntarism. American Journal of Psychiatry, 159, 705-712

Ryan, G.W. \& Bernard, H. R. (2000) Data management and analysis methods. In Handbook of Qualitative Research (eds N. K. Denzin \& Y. S. Lincoln), pp. 769-802. Thousand Oaks, CA: Sage.

Schoenwald, S. K. \& Hoagwood, K. (200I) Effectiveness, transportability, and dissemination of interventions: what matters when? Psychiatric Services, 52. $1190-1197$.
Sherman, P. S. (1998) Computer-assisted creation of psychiatric advance directives. Community Mental Health Journal, 34, 35I-362.

Slewchuk, T. R. (1998) Completing advance directives for health care decision: getting to yes. Psychology, Public Policy and Law, 4, 703-718.

Spradley, J. P. (1980) Participant Observation. New York: Rinehart \& Winston.

Srebnik, D. \& Brodoff, L. (2003) Implementing psychiatric advance directives: service provider issues and answers. Journal of Behavioral Health Services and Research, 30, 253-268.

Srebnik, D. S., Russo, J., Sage, J., et al (2003) Interest in psychiatric advance directives among higher users of crisis services and hospitalization. Psychiatric Services, 54 981-986.

Strauss, A. L. \& Corbin, J. (1990) Basics of Qualitative Research: Ground Theory Procedures and Techniques. Newbury Park, CA: Sage.

Swanson, J. W., Tepper, M. C., Backlar, P., et al (2000) Psychiatric advance directives: an alternative to coercive treatment? Psychiatry, 63, 160-172.

Swanson, J.W., Swartz, M. S., Hannon, M. J., et al (2003) Psychiatric advance directives: a survey of persons with schizophrenia, family members, and treatment providers. International journal of Forensic Mental Health, 2, 73-86.

Thomas, P. (2003) How should advance statements be implemented? British Journal of Psychiatry, 182, 548-549.

Valletto, N. M., Kamahele, R., Menon, A. S., et al (2002) Completion of advance directives for general health care among inpatients with schizophrenia. Journal of Nervous and Mental Disease, 190, 264-265.

Wendler, D., Martinez, R. A., Fairclough, D., et a (2002) Views of potential subjects toward proposed regulations for clinical research with adults unable to consent. American Journal of Psychiatry, 159, 585-591. 